\title{
Relationship between pre-existing kyphosis and new vertebral compression fractures following percutaneous vertebroplasty in patients with osteoporotic thoracolumbar fracture.
}

\section{Chao Chen}

Tongren Hospital Shanghai Jiaotong University School of Medicine https://orcid.org/0000-0002-49111655

Ronghua Yu

Department of Orthopedics, Tongren Hospital, Shanghai Jiao Tong University School of Medicine Liwei Chen

Tongren Hospital Shanghai Jiaotong University School of Medicine

\section{Silian Wang}

Tongren Hospital Shanghai Jiaotong University School of Medicine

Zhikun Li

Tongren Hospital Shanghai Jiaotong University School of Medicine

Youjia Xu ( $\nabla$ xuyoujia@suda.edu.cn )

https://orcid.org/0000-0002-9826-4324

Research article

Keywords: Aged; Osteoporosis; Spinal compression fractures; Digital imaging analysis; thoracolumbar ; Percutaneous vertebroplasty; Risk factor

Posted Date: March 9th, 2020

DOI: https://doi.org/10.21203/rs.3.rs-16288/v1

License: (a) This work is licensed under a Creative Commons Attribution 4.0 International License. Read Full License 


\section{Abstract}

Background: Thoracolumbar kyphosis disrupts the overall balance of the spine, thus the stress on each segment of the spine is different from that of the normal sequence. This may affect the outcome after Percutaneous vertebroplasty (PVP). But few studies have examined the relationship between thoracolumbar kyphosis and new fractures after PVP. Methods: A cross-sectional was conducted to evaluate the effect of the thoracolumbar kyphosis angle on postoperative recurrent fractures in patients with osteoporotic thoracolumbar fractures. From 2013 to 2018, 486 patients were treated with PVP in our hospital, and 242 patients were included in the study. These patients were divided into groups $A$ and $B$ based on the Cobb angle (greater or less than $10^{\circ}$ ) in the thoracolumbar region. Patients were followed up for 36 months. The average CT value of the thoracolumbar spine, BMD, and the time of recurrence of fractures were recorded. Results: A clear relationship was observed between thoracolumbar Cobb kyphosis and recurrent vertebral fractures $(O R=1.061, P=0.003)$. In group $A$, the proportion of women was larger and the BMD values was lower than that in group B. But no difference was found in the average $\mathrm{CT}$ value. K-M survival analysis found no significant difference in the rate of fracture recurrence between the two groups, but the time interval of second fracture occured at adjacent levels was significantly shorter than that occurred at distant levels in group $A$, which was not found in group B. Conclusions: When patients have thoracolumbar kyphosis before surgery, PVP should not be a recommended treatment.

\section{Background}

Osteoporotic thoracolumbar vertebral compression fractures are a common injury in middle-aged and elderly people and can lead to low back pain, decreased mobility, and spinal imbalance. One important factor of these complications is thoracolumbar kyphosis, which can be caused by compression of the vertebral body. Moreover, other conditions, such as degenerative discs, wedge deformation of the vertebral body, and Kummell's disease, which are prone to occur in patients with osteoporosis, can also increase thoracolumbar deformity ${ }^{[1]}$.

PVP is a minimally invasive conservative operation that can effectively relieve short-term pain caused by osteoporotic compression fractures and enable early out-of-bed activities ${ }^{[2]}$. Expansion of the vertebral body can temporarily increase the strength and restore the height of the body; however, this effect cannot be maintained for a long time, and the procedure can cause many complications as well ${ }^{[3,4]}$. New compression fracture is one of the most common complications after PVP. Many studies have reported risk factors for new fractures after PVP, but few studies have analyzed the relationship between thoracolumbar kyphosis and new fractures. It is unclear whether spinal imbalance is a risk factor for vertebral compression fractures ${ }^{[5]}$. In this study, we retrospectively analyzed the effects of preoperative thoracolumbar kyphosis on new fractures after PVP.

\section{Methods}




\section{Patient selection and grouping}

From 2013 to 2018, a total of 486 patients underwent PVP in our hospital. All patients were older than 60 years old and had vertebral compression fracture of at least one segment from T10 to L3 confirmed by MRI. The exclusion criteria were as follows: 1) high-energy injuries (a shift of the center of gravity of the body exceeding half of the body height during injury or traffic accident injuries); 2)motor dysfunction of lower limbs; 3) postoperatively confirmed multiple myeloma (MM), ankylosing spondylitis (AS), metastatic bone tumors, rheumatoid and rheumatoid arthritis diseases (e.g., gout); and 4) a history of open reduction and internal fixation of the spine. This study was approved by the ethical committee of Shanghai Tongren Hospital.

All patients received anti-osteoporosis treatment and were followed up for a maximum of three years after the first operation. Follow-up was terminated if patients had recurrent thoracolumbar compression fractures.

\section{Surgical procedure}

Under local anesthesia using $1 \%$ lidocaine, the patient was situated in the prone position. All steps were completed using a C-arm machine. An 11-G bone marrow biopsy needle (REF T05E kyphx OsteoIntroducer System, kyphon Inc. Medtronic, Minnesota) was fixed, a working cannula was used to puncture the collapsed vertebral body through the pedicles of either side, and then the needle was inserted into the anterior one-third of the vertebral body under fluoroscopic guidance (Arcardis Varic mobile X-ray system, Siemens, Germany). After transpedicular positioning, the needle was exchanged over a guidewire for a working cannula (Kynefyc systems, Shanghai, China). During fluoroscopy, bone cement was injected through the working cannula until the filling material reached the posterior $1 / 3$ of the vertebral body or leakage of bone cement was observed on fluoroscopy. Patients were not allowed to perform out-of-bed activities after operation.

\section{Detection of parameters}

T-score: T-scores of the lumbar spine and the hip were determined by dual energy $\mathrm{X}$-ray absorptiometry (DXA) (Hologic Discovery, WI, Bedford, MA, USA) in all patients before the operation.

Hounsfield unit (HU) average value: All patients underwent MDCT (Light Speed pro VCT 32, GE, Buckinghamshire, UK) in the supine position one day before PVP. The scanning range was T10 to L5. All imaging data were transferred to the PACS system. The HU value was determined in the cancellous region of each vertebral body at the center of the sagittal plane of the vertebral body (usually at the level of the spinous process) by two spine surgeons according to the method described by Hao et al[6]. The cortical bone was avoided when selecting the detection area. The values independently measured by two investigators and then were averaged to determine the $\mathrm{HU}$ value of each vertebra. The final value was the average $\mathrm{HU}$ value of all vertebrae (T10-L5), except for the fractured vertebra. 
Thoracolumbar Cobb angle: All patients underwent a thoracolumbar X-ray examination in the anteroposterior standing position before surgery. The Cobb angle was measured from T10 to L2. A positive Cobb angle was recorded as lordosis, and a negative angle was recorded as convex. According to the Cobb angle of the Thoracolumbar junction (TLJ), the subjects were divided into two groups: Group $A$ $\left(\mathrm{TLJ}>10^{\circ}\right)$ and Group B $\left(\mathrm{TLJ}<10^{\circ}\right)$.

\section{Statistical analysis}

Independent Student's $t$ tests, Mann-Whitney $U$ tests, Chi-square tests and correlation analysis were used for comparisons between group A and group B. K-M survival analysis was used to compare the occurrence time of refracture between the two groups. A Cox regression model was used to analyze the relationship between refracture and age, thoracolumbar Cobb angle, bone mineral density (BMD) and HU value. Odds ratios were also calculated. A value of $P<0.05$ was considered as a statistically significant difference in all tests. Statistical Package for the Social Sciences software version 22.0 (SPSS, Inc., Chicago, IL) was used for the data analysis.

\section{Results}

Among all 486 patients, 242 patients ( 61 males and 181 females) were enrolled according to the inclusion criteria, with a BMD of $(-2.07 \pm 1.18)$. Based on the thoracolumbar kyphosis angle in the standing position before surgery, 126 patients ( 23 males and 103 females) were included in Group $A$, and 116 patients (38 males and 78 females) were included in Group B. Statistically significant differences were found in the male to female ratio and T-score of bone density between the two groups, while other parameters showed no significant differences (Table 1). A total of 277 vertebrae were treated with primary PVP (154 segments in Group A and 123 segments in Group B). Table 2 shows the distribution of vertebrae treated by PVP in both groups. All patients were followed up for $(13.53 \pm 8.49)$ months on average (range 1-36 months). Follow-up was terminated if the patient suffered new fractures, as previously mentioned. Fifty-five patients (65 vertebrae) experienced fracture of new segments at an average of 225 days (range 12-715 days) after the first operation and subsequently underwent second PVP. The K-M analysis (Fig. 1) showed that a preoperative Cobb angle $\geq 10^{\circ}$ of the thoracolumbar segments did not affect the probability of refracture. However, the regression analysis indicated that thoracolumbar Cobb kyphosis was significantly associated with vertebral refracture $(O R=1.061, P=0.003)$ (Table 4). In Group A, the time interval to second fracture for adjacent vertebrae was significantly shorter than that for distant vertebrae. This phenomenon was not found in Group B (Table 3).

\section{Discussion}

PVP was once extensively recommended applied for conservative treatment of osteoporosis vertebral compression fractures. This method has the advantages of simple operation, rapid pain relief, and reduced suffering of patients ${ }^{[7]}$, but it shows no superiority in maintaining vertebral height ${ }^{[8.9]}$. 
Fracture of new vertebral segments is a common complication after vertebral expansion, with an incidence of $15 \%$ during the first postoperative year ${ }^{[10]}$. In the literature, the risk factors for new fractures include BMI, age, gender, osteoporosis, bone cement injection, cement leakage, a history of vertebral fracture and use of steroid drugs ${ }^{[8,11,12]}$. In particular, cement leakage, bone mineral density and vertical height restoration are significantly related to the incidence of fracture of adjacent vertebrae ${ }^{[13]}$. In our study, the longest follow-up period was 3 years, and the incidence of new vertebral fractures was $22 \%$. Spinal imbalance can be easily ignored in clinical practice because patients with spinal imbalance often have other widely accepted risk factors that may cause osteoporotic vertebral compression fractures. In our study, Group A had a higher proportion of women, a lower BMD, and more cases of osteoporotic fractures. These factors are widely reported as being associated with osteoporotic compression fractures/refractures ${ }^{[10,12]}$. It is difficult to exclude these confounding factors in comparative experiments.

Height restoration of a vertebra by single vertebral expansion can improve the compression of the vertebra itself, but the overall balance cannot be easily restored. Moreover, the restored vertebral height after PVP cannot be effectively maintained, and a risk of collapse of the upper and lower endplates of the vertebra happens occasionally ${ }^{[14]}$.

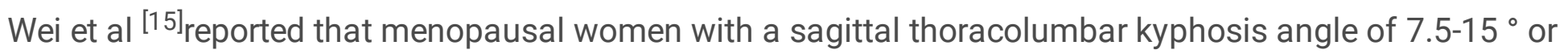
thoracolumbar kyphosis angle $>15^{\circ}$ have a higher risk of vertebral compression fractures. Movrin et al [16] found that after percutaneous balloon-assisted kyphoplasty (PKP) or PVP, if the thoracolumbar kyphotic angle was still larger than $9^{\circ}$ and the BMD was greater than or equal to 3.0, there was a higher probability of refracture of adjacent segments.

The regression analysis in our study showed that the thoracolumbar kyphosis angle was correlated with vertebral refracture in both univariate and multivariate Cox regression. In addition, the imaging findings demonstrated that the mean CT-value, reflecting the overall cancellous bone content of the thoracolumbar spine, was significantly correlated with vertebral refracture, while BMD value was not correlated with vertebral refracture under any circumstance.

Many studies have reported that overcorrection of the vertebra is a cause of new fractures ${ }^{[9.13,17]}$. Excessive height restoration of the compressed and fractured vertebra may lead to overexpansion of the vertebral endplates, which produces pressure on adjacent unstable vertebrae through the intervertebral disc, thus causing new compression fractures of adjacent vertebrae ${ }^{[18]}$. Deibert et al ${ }^{[19]}$ found that fracture of adjacent vertebrae occurred earlier than that of distant vertebrae after vertebral expansion surgery. These findings have also been reported by other researchers ${ }^{[8,20,21]}$. In our study, no significant difference in the restoration of vertebral height was found between Group A and Group B after PVP; the time to new fracture of adjacent vertebrae after PVP was significantly shorter than that for distant vertebrae in Group A, while this phenomenon was not found in Group B. We hypothesized that a spinalpelvic imbalance exists in patients with thoracolumbar kyphosis; vertebral expansion surgery changes the modulus of elasticity/stiffness of one or more vertebrae in the spinal sequence, resulting in a change in 
the stress on adjacent vertebrae. This effect may be magnified in patients with spinal imbalance. Meanwhile the tension in the surrounding muscle and soft tissue is higher than that in patients with a normal spine sequence, and thus new fracture of adjacent vertebrae occurs earlier.

In this study, case inclusion criteria were based on the force during injury, instead of BMD, because although osteoporotic patients have a higher risk of thoracolumbar compression fractures, this does not mean that all patients with reduced BMD and compression thoracolumbar fracture should be diagnosed with osteoporotic fracture. Lumbar degenerative scoliosis may also cause deviations in BMD measurement results and is also very common in patients with osteoporosis.

The limitation of this study is the baseline difference between Group A and Group B. Additional multicenter prospective paired analyses should be conducted to further reduce the influence of confounding factors.

\section{Conclusion}

PVP is not recommended for patients with thoracolumbar kyphosis before surgery.

\section{List Of Abbreviations}

BMD: Bone mineral density

PVP: Percutaneous vertebroplasty

PKP: Percutaneous balloon-assisted kyphoplasty

HU: Hounsfield unit

MM: multiple myeloma

AS: ankylosing spondylitis

TLJ: Thoracolumbar junction

DXA: Dual-energy X-ray absorptiometry

CT: Computed tomography

MDCT: Multislice spiral CT

SPSS: Statistical product and service solutions

OR: Odds ratios

\section{Declarations}




\section{Ethics approval and consent to participate}

The experimental protocol was established, according to the ethical guidelines of the Helsinki Declaration and was approved by the Human Ethics Committee of Tongren Hospital, Shanghai Jiao Tong University School of Medicine. Written informed consent was obtained from individual or guardian participants

\section{Availability of data and materials}

The datasets used and/or analysed during the current study are available from the corresponding author on reasonable request.

\section{Competing interests}

The authors declare that they have no competing interests.

\section{Funding}

1. Suzhou people's livelihood science and technology project. Award Number: SS201814 | Recipient: Youjia Xu, Ph.D.

\section{Authors' contributions}

CC, YRH conceived and designed the study. CLW and WSL measure and record the data. LZK wrote the paper. XYJ reviewed manuscript and revised the manuscript critically for important intellectual content. All authors read and approved the manuscript.

\section{Acknowledgements}

Not applicable.

\section{References}

[1] Xia W, Fu H, Zhu Z, et al. Association between back muscle degeneration and spinal-pelvic parameters in patients with degenerative spinal kyphosis. BMC Musculoskelet Disord. 2019; 20(1): 454.

[2] Jensen ME, Evans AJ, Mathis JM, Kallmes DF, Cloft HJ, Dion JE. Percutaneous polymethylmethacrylate vertebroplasty in the treatment of osteoporotic vertebral body compression fractures: technical aspects. AJNR Am J Neuroradiol. 1997; 18(10): 1897-904.

[3] Trout AT, Kallmes DF, Lane JI, Layton KF, Marx WF. Subsequent vertebral fractures after vertebroplasty: association with intraosseous clefts. AJNR Am J Neuroradiol. 2006; 27(7): 1586-91.

[4] Uppin AA, Hirsch JA, Centenera LV, Pfiefer BA, Pazianos AG, Choi IS. Occurrence of new vertebral body fracture after percutaneous vertebroplasty in patients with osteoporosis. Radiology. 2003; 226(1): 119-24. 
[5] Baek SW, Kim C, Chang H. The relationship between the spinopelvic balance and the incidence of adjacent vertebral fractures following percutaneous vertebroplasty. Osteoporos Int. 2015; 26(5): 1507-13.

[6] Chen HJ, Xiao ZG, Yu RH, Wang Y, Xu RJ, Zhu XD. CT measurement and analysis of the target vertebral body in elderly patients with uncompressed osteoporotic thoracolumbar fractures. Eur Rev Med Pharmacol Sci. 2018; 22 Suppl 1: 36-44.

[7] Chen AT, Cohen DB, Skolasky RL. Impact of nonoperative treatment, vertebroplasty, and kyphoplasty on survival and morbidity after vertebral compression fracture in the medicare population. $J$ Bone Joint Surg Am. 2013; 95(19): 1729-36.

[8] Yi X, Lu H, Tian F, et al. Recompression in new levels after percutaneous vertebroplasty and kyphoplasty compared with conservative treatment. Arch Orthop Trauma Surg. 2014; 134(1): 21-30.

[9] Chen LH, Hsieh MK, Liao JC, et al. Repeated percutaneous vertebroplasty for refracture of cemented vertebrae. Arch Orthop Trauma Surg. 2011;131(7): 927-33.

[10] Rho YJ, Choe WJ, Chun YI. Risk factors predicting the new symptomatic vertebral compression fractures after percutaneous vertebroplasty or kyphoplasty. Eur Spine J. 2012; 21(5): 905-11.

[11] Cao J, Kong L, Meng F, Zhang Y, Shen Y. Risk factors for new vertebral compression fractures after vertebroplasty: a meta-analysis. ANZ J Surg. 2016; 86(7-8): 549-54.

[12] Ren HL, Jiang JM, Chen JT, Wang JX. Risk factors of new symptomatic vertebral compression fractures in osteoporotic patients undergone percutaneous vertebroplasty. Eur Spine J. 2015; 24(4): 7508.

[13] Ma X, Xing D, Ma J, et al. Risk factors for new vertebral compression fractures after percutaneous vertebroplasty: qualitative evidence synthesized from a systematic review. Spine (Phila Pa 1976). 2013; 38(12): E713-22.

[14] Chou KN, Lin BJ, Wu YC, Liu MY, Hueng DY. Progressive kyphosis after vertebroplasty in osteoporotic vertebral compression fracture. Spine (Phila Pa 1976). 2014; 39(1): 68-73.

[15] Wei Y, Tian W, Zhang GL, Lv YW, Cui GY. Thoracolumbar kyphosis is associated with compressive vertebral fracture in postmenopausal women. Osteoporos Int. 2017; 28(6): 1925-1929.

[16] Movrin I, Vengust R, Komadina R. Adjacent vertebral fractures after percutaneous vertebral augmentation of osteoporotic vertebral compression fracture: a comparison of balloon kyphoplasty and vertebroplasty. Arch Orthop Trauma Surg. 2010; 130(9): 1157-66.

[17] Lin CC, Chen IH, Yu TC, Chen A, Yen PS. New symptomatic compression fracture after percutaneous vertebroplasty at the thoracolumbar junction. AJNR Am J Neuroradiol. 2007; 28(6): 1042-5. 
[18] Yoo CM, Park KB, Hwang SH, Kang DH, Jung JM, Park IS. The analysis of patterns and risk factors of newly developed vertebral compression fractures after percutaneous vertebroplasty. J Korean Neurosurg Soc. 2012; 52(4): 339-45.

[19] Deibert CP, Gandhoke GS, Paschel EE, Gerszten PC. A Longitudinal Cohort Investigation of the Development of Symptomatic Adjacent Level Compression Fractures Following Balloon-assisted Kyphoplasty in a Series of 726 Patients. Pain Physician. 2016; 19(8): E1167-E1172.

[20] Mazzantini M, Carpeggiani P, d'Ascanio A, Bombardieri S, Di Munno O. Long-term prospective study of osteoporotic patients treated with percutaneous vertebroplasty after fragility fractures. Osteoporos Int. 2011; 22(5): 1599-607.

[21] Takahara K, Kamimura M, Moriya $\mathrm{H}$, et al. Risk factors of adjacent vertebral collapse after percutaneous vertebroplasty for osteoporotic vertebral fracture in postmenopausal women. BMC Musculoskelet Disord. 2016; 17: 12.

\section{Figures}

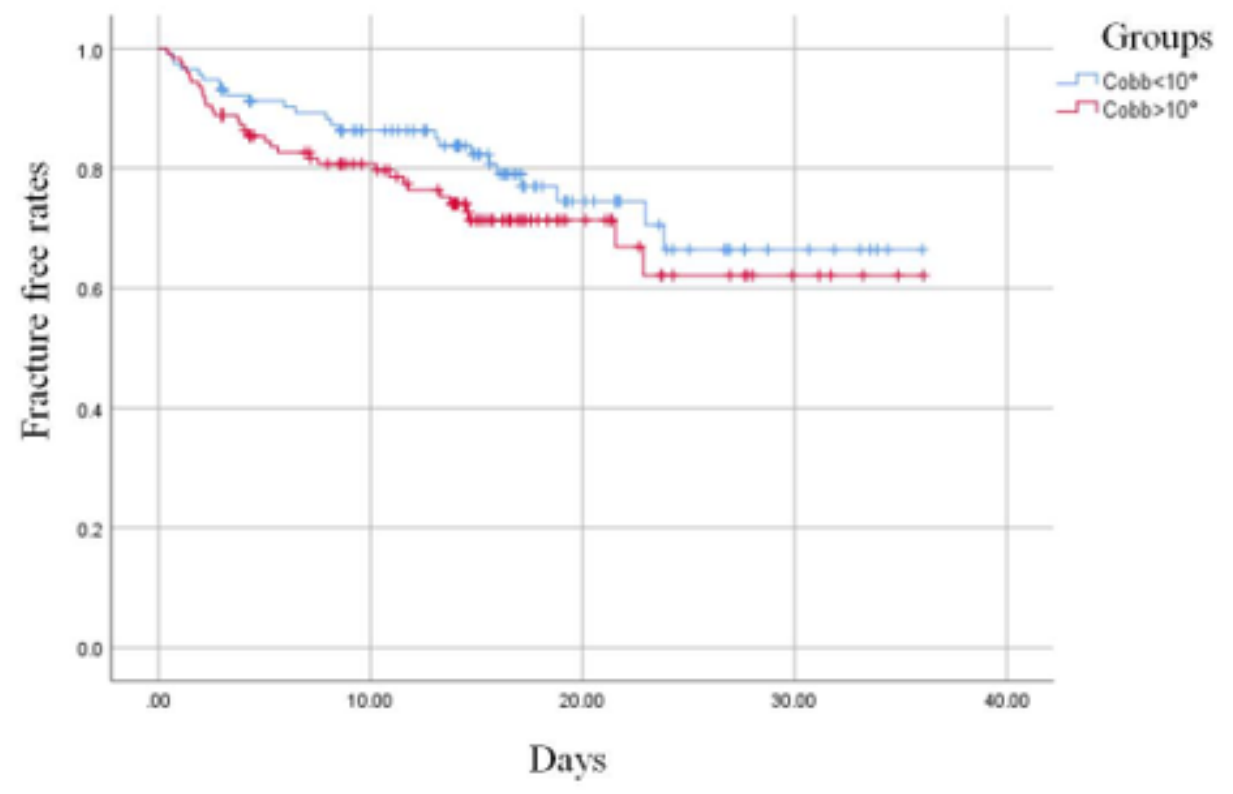

In Group A, Cobb angle $>10^{\circ}$; in Group $\mathrm{B}, \mathrm{Cobb}$ angle $<10^{\circ} ; P=0.198$

\section{Figure 1}

The rate of fracture free over time 